\title{
Aspectos psicosociales de los programas de salud de personas mayores con hipoacusia y su impacto en la adherencia al uso de audífonos: una revisión narrativa
}

\section{Psychosocial aspects of health programs for elderly people with hearing loss and its impact on hearing aid adherence: a narrative review}

Felipe Cardemil M. ${ }^{1,2}$, Cristina Sade B. ${ }^{3}$, Eduardo Fuentes L. ${ }^{4}$, Graciela Rojas C. $^{5}$

Departamento de Otorrinolaringología, Facultad de Medicina, Universidad de

Chile. Santiago, Chile.

${ }^{2}$ Departamento de

Otorrinolaringología, Clínica

Las Condes. Santiago, Chile.

${ }^{3}$ Unidad de Psicooncología, Instituto Nacional del Cáncer,

Chile. Santiago, Chile.

${ }^{4}$ Carrera de Fonoaudiología,

Departamento de Ciencias

de la Salud, Facultad

de Medicina, Pontificia Universidad Católica de Chile. Santiago, Chile.

${ }^{5}$ Departamento de Psiquiatría y Salud Mental, Facultad de Medicina, Universidad de Chile, Hospital Clínico de la Universidad de Chile. Santiago, Chile.

Los autores declaran no tener conflictos de interés

Recibido el 23 de junio de 2020. Aceptado el 2 de septiembre de 2020

Correspondencia: Felipe Cardemil M.

Departamento de Otorrinolaringología Facultad de Medicina, Universidad de Chile.

Santiago, Chile.

Email: felipecardemil@med. uchile.c

\section{Resumen}

El envejecimiento progresivo de la población mundial es una preocupación reconocida por la mayoría de las propuestas de políticas públicas en diversas áreas, incluida salud. La persona mayor pertenece a un grupo social vulnerable, que requiere ser considerado en las intervenciones y políticas de salud pública. Existe una considerable pérdida de las capacidades sensoriales y motrices que suponen una disminución de la autonomía, la que si se asocia a la realidad de las personas mayores con menos ingresos, dificulta un acceso oportuno a prestaciones de salud para poder enfrentar la discapacidad de manera apropiada. Las consecuencias de la pérdida de la capacidad auditiva en la persona mayor se manifiestan en problemas de la comprensión del habla, deterioro cognitivo y trastornos de la salud mental como ansiedad y depresión. Lamentablemente, las intervenciones para aumentar la adherencia de los usuarios al uso de audífonos no han mostrado muchos resultados favorables. Un correcto diagnóstico permite tener una orientación y rehabilitación adecuada a través de la generación de programas y estrategias enfocadas en la integración social, permitiendo que la calidad de vida se mantenga o mejore no solo en las personas con hipoacusia. Implementar un abordaje que busque tener un impacto positivo en la adherencia al uso de audífonos debe incluir y desarrollar programas de rehabilitación auditiva que consideren aspectos de contexto como vivienda, red de apoyo e integración social, entre otros.

Palabras clave: Hipoacusia, envejecimiento, aspectos psicosociales, rehabilitación.

\begin{abstract}
Progressive aging of the world population is a concern recognized by most public policy proposals in various areas, including health. The elderly belongs to a vulnerable social group, which requires consideration in public health interventions and policies. There is a considerable loss of sensory and motor capacities that decreases autonomy, which if associated with lesser income in older adults, hinders timely access to health benefits and its possibility to face disability appropriately. The consequences of hearing loss in the elderly are manifested in problems of speech comprehension, cognitive deterioration, and mental health disorders such as anxiety and depression. Unfortunately, interventions to increase user adherence to hearing aid use have not shown many positive results. A correct diagnosis allows to have an adequate orientation and rehabilitation through the generation of programs and strategies focused on social integration, allowing the quality of life to be maintained or improved not only in people with hearing loss. Implementing an approach that seeks to have a positive impact on adherence to hearing aid use should include and develop auditory rehabilitation programs that consider contextual aspects such as housing, support network and social integration, among others.
\end{abstract}

Keywords: Hearing loss, aging, psychosocial aspects, rehabilitation. 


\section{Introducción}

El envejecimiento progresivo de la población mundial es una preocupación reconocida por la mayoría de las propuestas de políticas públicas en diversas áreas, incluida salud. Cerca del 23\% de las personas de países desarrollados y el 9\% de las personas de países en vías de desarrollo tienen 60 años o más, cifras que tienden al alza en proyecciones que para el año 2100 esperan un 34\% y un 27\%, respectivamente ${ }^{1}$. A nivel latinoamericano, el envejecimiento durante esta década incluye 43 personas de 65 años o más por cada 100 personas menores de 15 años ${ }^{2}$.

El progresivo envejecimiento de la población mundial convierte a la "persona mayor" en objeto de intervenciones sanitarias. Vinculado a la actividad laboral y la edad de jubilación, este concepto clasifica a las personas de acuerdo a un corte etario que en la mayoría de los países recae en el límite de 65 años de edad. Las acciones destinadas a evitar la progresión del deterioro de las capacidades funcionales del cuerpo, producto de los años o de factores externos, se ven intensificadas a partir de dicha edad. El concepto de la persona mayor consolida aquella relación entre factores sociales vinculados a la mayor edad (ej. actividad laboral e ingreso económico) y los procesos biológicos del deterioro de la salud.

La estrategia nacional para el cumplimiento de los objetivos sanitarios de la década 20102020 señala que las personas mayores "pueden presentar múltiples enfermedades crónicas, de tal manera que el status de enfermo es permanente $y$, en la mayoría de los casos, definitivo"3. A partir de esto, la estrategia es, entonces, mantener la funcionalidad en las personas de este grupo, aquella capacidad de realizar determinadas acciones requeridas en el diario vivir. En este esfuerzo resulta importante el abordaje de la hipoacusia, condición que resulta incapacitante en la mayoría de los casos y que se presenta con mayor frecuencia a edades más avanzadas ${ }^{4}$.

\section{La hipoacusia como problema de la persona mayor}

La pérdida de la capacidad auditiva no es un problema aislado, al contrario, resulta bastante frecuente y sus proyecciones van en alza con- siderando su relación con el envejecimiento. La Organización Mundial de la Salud (OMS) estimó que para el año 2014 serían cerca de 360 millones de personas las que en el mundo vivirían con hipoacusia que generaría algún tipo de discapacidad (hipoacusia en rango moderado $)^{5}$, esperando que para el año 2025, producto del envejecimiento de la población, la cifra aumentaría a más de mil millones ${ }^{6}$.

Considerada tradicionalmente como una condición natural y propia del envejecimiento, se estima que la audición, después de los 60 años de edad, disminuya en promedio $1 \mathrm{~dB}$ por año, con una pérdida observada mayor en hombres que en mujeres ${ }^{7}$. La prevalencia de personas con pérdida de capacidad auditiva ha sido a menudo subestimada debido a la lenta progresión de esta condición. En tal contexto, la hipoacusia resulta una condición de salud subdiagnosticada y subtratada ${ }^{8}$. La estimación del $15 \%$ de la población mundial adulta con algún grado de hipoacusia se eleva a poco más del $30 \%$ cuando solamente se considera la población mayor de 65 años y en un $60 \%$ en mayores de 85 años 9 .

Las cifras referentes a la realidad chilena sostienen que $91,1 \%$ de los mayores de 80 años presentaban algún grado de hipoacusia de acuerdo a la Encuesta Nacional de Salud del año $2003^{10}$. La versión de esta encuesta realizada en el periodo 2009-2010 estimó que un $52,4 \%$ de las personas de 65 años o más presentaban esta condición en algún grado ${ }^{11}$. La importancia de las cifras de prevalencia, como de las tendencias al envejecimiento, se justifican por la relación existente entre la disminución de la capacidad auditiva y el envejecimiento. Tal relación ha llevado a definir un tipo de alteración sensorial propia para este grupo etario. La "presbiacusia" es definida como aquella disminución de la capacidad auditiva vinculada al envejecimiento de las personas, que actualmente prefiere denominarse "hipoacusia asociada al envejecimiento"12.

El inicio de la disminución de la audición en la adultez mayor considera una sumatoria de factores extrínsecos e intrínsecos que producen cambios en las estructuras auditivas centrales y periféricas propias del proceso de envejecimiento ${ }^{13}$. Con un inicio a partir de los 20 a 30 años de edad y generando sus primeras manifestaciones a los 50 a 60 años de edad, la 
presbiacusia constituye un fenómeno biológico del que ninguna persona puede escapar ${ }^{14}$. Existen diferencias por sexo; los hombres son quienes más frecuentemente padecen esta condición, situación atribuida a la mayor exposición a ambientes ruidosos ${ }^{14}$. Además, los factores que se asocian al riesgo de hipoacusia producto del envejecimiento pueden ser modificables y no modificables, clasificándose en cuatro categorías: envejecimiento coclear (edad); causas ambientales (exposición ocupacional y recreacional a ruido, exposición a fármacos ototóxicos); predisposición genética (sexo, raza, alteraciones genéticas) y comorbilidades (hipertensión, diabetes, eventos cardiovasculares, tabaquismo $)^{5}$.

\section{Consecuencias de la pérdida auditiva asociada al envejecimiento}

Las consecuencias de la pérdida de capacidad auditiva en la persona mayor se manifiestan también en problemas de la comprensión del habla, especialmente cuando existe ruido de fondo o reverberación ${ }^{15}$. Los problemas de comunicación oral, entendida como la habilidad para participar activa y efectivamente de una conversación en distintos medios de escucha, se asocian con una reducción en la calidad de vida, tanto en quien padece esta dificultad como en su entorno ${ }^{16}$. Dichos problemas representan una de las principales razones de búsqueda de ayuda médica por parte de los adultos mayores con hipoacusia.

Considerando la importancia de la comunicación oral y las dificultades observadas en las personas mayores, el "Committee on Hearing, Bioacoustics and Biomechanics" propuso en 1988 un modelo acerca de los aspectos que afectan adversamente la comunicación oral en las personas mayores ${ }^{17}$. De acuerdo a este modelo, los cambios relacionados al envejecimiento de las estructuras auditivas periféricas y centrales, así como la disminución de las funciones cognitivas, tales como memoria y atención, aisladas o en combinación entre ellas, llevan consigo dificultades en la comunicación oral.

Otras dificultades asociadas a la hipoacusia son los cuadros de deterioro cognitivo como la demencia ${ }^{18}$, y trastornos de la salud mental como ansiedad y depresión ${ }^{8,19,20}$. Las repercusiones en torno a las dificultades de comunicación podrían tener sus efectos en la relación entre la hipoacusia y lo que Mick y $\mathrm{Lin}^{21}$ señalan como aislamiento social manifestado en la presencia o ausencia de apoyo emocional, apoyo financiero, amigos cercanos, de cónyuge o de pareja. El impacto de la hipoacusia en los aspectos de la vida cotidiana hacen que, tanto la satisfacción por la vida y en general los aspectos psicosociales, debieran ser enfatizados como una parte cotidiana de la rehabilitación auditiva ${ }^{22}$. Las implicancias físicas y sociales que desencadena la hipoacusia en la población adulta mayor dan cuenta de la necesidad de desarrollar intervenciones para revertir su impacto en la calidad de vida de las personas. Dichas iniciativas deberán considerar que estamos frente a un problema "propio del envejecimiento" y que debieran contemplar una intervención integral incluyendo los estilos de vida.

\section{La discapacidad y sus consecuencias en el envejecimiento}

Según la “Comisión Económica para América Latina y el Caribe" (CEPAL) se estima que un $12,4 \%$ de la población en América Latina vive con algún grado de discapacidad ${ }^{23}$. La hipoacusia se enmarca dentro de las discapacidades, concepto cargado de aspectos negativos, en tanto es una deficiencia de la capacidad para realizar una actividad en forma y dentro del margen que se considera normal para un ser humano en su contexto social, restringiendo la vinculación de la persona con su entorno. La discapacidad se vincula directamente con la calidad y expectativas de vida de quienes la padecen, ya que estas personas se ven enfrentadas a limitación y restricciones tanto por problemas de salud, personales e incluso ambientales como la falta de apoyo social ${ }^{24}$.

La hipoacusia ocupa el tercer lugar entre las patologías que involucran años de vida con discapacidad (years lived with disability, YLDs) luego de la depresión y lesiones accidentales 5 . La población mundial que sufre de algún grado de hipoacusia que genera discapacidad es cercana al 5,3\% ${ }^{9}$. Esta condición, además, tiene importantes consecuencias para el de- 
sarrollo de los países debido a que disminuye la productividad de quienes la padecen, así como también se requiere de una educación especializada, al igual que mayores prestaciones de salud ${ }^{25}$.

La discapacidad por hipoacusia tiene consecuencias como limitar el establecer relaciones sociales interpersonales y en la participación en actividades grupales ${ }^{26}$. La hipoacusia afecta la calidad de vida de quienes la padecen, en particular el funcionamiento psicológico, social y emocional ${ }^{27}$. Como se mencionó previamente, se ha vinculado con condiciones psicológicas entre las que se destaca la depresión, soledad, ansiedad, somatización y funcionamiento social pobre ${ }^{27}$. La depresión constituye la enfermedad psiquiátrica más frecuentemente relacionada con hipoacusia en adultos ${ }^{28}$.

Se han realizado otras vinculaciones de carácter social en torno a la discapacidad producto de la hipoacusia. Es así como estudios le atribuyen un impacto socioeconómico al observar que usuarios hipoacúsicos tienen mayores chances de recibir salarios más bajos o bien tener mayor posibilidad de permanecer desempleados ${ }^{29}$. Desde la óptica del "modelo de determinantes sociales" postulado por la OMS, existiría vulnerabilidad diferencial para este grupo social -constituyéndose como un grupo vulnerable- lo que podría estar mediando el efecto que tiene la posición social de las personas con discapacidad auditiva y los resultados en salud. Las consecuencias sociales y económicas de la discapacidad, a su vez, podrían retroalimentar los mecanismos, contribuyendo al desarrollo de otras enfermedades ${ }^{30}$.

La discapacidad es una condición que cualquier persona pudiese llegar a padecer, pero existen grupos etarios donde predomina. El adulto mayor ocupa un $43,4 \%$ del total de personas con alguna discapacidad, es decir, 2 de cada 5 adultos mayores la presentan, observando una mayor prevalencia en discapacidades visuales, seguidas por discapacidad física y auditiva ${ }^{31}$. La persona mayor pertenece a un grupo social vulnerable, que requiere ser considerado en las intervenciones y políticas de salud pública, ya que es sabido que a medida que avanzamos en edad la capacidad funcional se ve disminuida. Existe una considerable pérdida de las capacidades sensoriales y motrices que suponen una disminución de la autono- mía, y si se considera el ámbito económico de la persona mayor, habitualmente con ingreso menor, ella presenta un acceso restringido para poder enfrentar la discapacidad de manera apropiada, pudiendo así elevar el grado de discapacidad por las bajas posibilidades en términos de tratamiento o apoyo. Esto resulta especialmente relevante en un país como Chile dado el alto nivel de inequidad en el ingreso que presenta ${ }^{32}$, el que incluso es elevado para los estándares de América Latina, una de las regiones más inequitativas en el mundo ${ }^{32}$. Se ha observado que determinados grupos vulnerables-como lo son las personas mayores-, experimentan peores condiciones de salud en entornos más desiguales ${ }^{33}$.

El informe mundial sobre la discapacidad de la $\mathrm{OMS}^{34}$, plantea la necesidad de una rehabilitación basada en la comunidad, la que permitiría "abordar los obstáculos que tienen las personas con discapacidad para acceder a la atención en salud", resultando satisfactoria en contextos de pocos recursos. Con lo anterior se facilitaría el acceso de las personas con discapacidad a los servicios existentes, evaluando y promoviendo los servicios sanitarios preventivos, posibilitando una intervención temprana complementada con derivaciones a servicios secundarios. Esta modalidad también puede "mejorar aptitudes y actitudes, reforzar la formación en el empleo y proporcionar orientación a los empleadores" 34 .

Según el mencionado informe, invertir en rehabilitación permite "promover el funcionamiento de las personas con múltiples problemas de salud, junto con la entrega de servicios lo más cerca posible de donde las personas viven". En esa misma línea estaría el integrar la rehabilitación en los establecimientos de atención primaria y secundaria de salud con el objetivo de mejorar la disponibilidad. En relación a las ayudas técnicas auxiliares, el informe aboga por el aumento en el acceso, lo que podría mejorar la autonomía, fomentar la participación y reducir los costos de asistencia y apoyo ${ }^{34}$.

\section{El manejo de la hipoacusia y el problema de la adherencia terapéutica}

El manejo de la hipoacusia puede ser dividido en dos grandes grupos: aquellos programas de rehabilitación a través de consejerías 
y educación, y los que incluyen la utilización de dispositivos de ayuda auditiva, dentro de los que se incluyen los audífonos, dispositivos de asistencia auditiva y los implantes cocleares $^{21,35}$. La efectividad de estas intervenciones dependerá en parte si se trata de un daño periférico del sistema auditivo o bien a nivel central. Ejemplo de aquello es lo propuesto por Humes $^{36}$, al señalar que si las dificultades en la comunicación oral del adulto mayor resultan de cambios en las estructuras auditivas centrales, entonces probablemente la adaptación protésica no muestre beneficios significativos en la persona. Los signos de los cambios en las estructuras periféricas de la audición son bastante $\operatorname{conocidos}^{37}$, no así aquellos que se producen en las estructuras auditivas centrales, restringiendo las intervenciones de rehabilitación en las personas mayores.

Las ayudas auditivas externas, como los audífonos, resultan ser la opción de tratamiento de mayor disponibilidad para quienes sufren de pérdida auditiva relacionada al envejecimiento ${ }^{38}$. El uso de estos dispositivos ha aumentado exponencialmente, estimando que en EE. UU. su utilización se ha incrementado en casi un 300\% durante el decenio comprendido entre 1996 y $2006^{39}$. Sin embargo, aún existen desafíos en el acceso, considerando que actualmente se fabrica solamente el 10\% de la necesidad global de audífonos.

El uso de audífonos en las personas mayores se fundamenta en los positivos resultados que tendrían en relación a la menor ansiedad y depresión ${ }^{40}$ incluso comparado con la población geriátrica general ${ }^{22}$, al mejoramiento de niveles auditivos globales y específicos de calidad de vida en personas con hipoacusia ${ }^{41}$, y a la disminución de consecuencias psicológicas, sociales y emocionales de la hipoacusia luego de la implementación con audífonos ${ }^{42}$. Estudios que evalúan el uso de estas tecnologías establecen que sólo un $20 \%$ de las personas mayores con hipoacusia moderada a profunda se perciben a sí mismos con algún grado de discapacidad. Por otro lado, solo un 25\% de los usuarios que cumplen los criterios para el uso de audífonos obtienen uno, mientras que un porcentaje importante de estos usuarios, teniendo un audífono, no lo utilizan ${ }^{24,43}$. La OMS señala que, mundialmente, solamente 1 de cada 5 personas que potencialmente se podrían beneficiar del uso de audífonos tienen acceso a estos ${ }^{44}$. Otros autores mencionan que solo 1 de cada 8 adultos con indicación de audífonos acepta su uso, y que el 85\% de los usuarios lo usa correctamente ${ }^{45}$.

La edad de implementación de audífonos en personas con hipoacusia es en promedio de 74 años, aun cuando los síntomas de hipoacusia se manifiesten desde años antes ${ }^{46}$. Asimismo, entre el 30\% y 45\% de los adultos mayores que reciben audífonos por primera vez no los utilizan ${ }^{43}$. Estos antecedentes hacen de la adherencia a los tratamientos un concepto importante en el manejo de la hipoacusia en personas mayores y una permanente preocupación entre los profesionales sanitarios.

La adherencia a los tratamientos es definida por la OMS como el "El grado en que el comportamiento de una persona -tomar el medicamento, seguir un régimen alimentario y ejecutar cambios del modo de vida- se corresponde con las recomendaciones acordadas de un prestador de asistencia sanitaria" ${ }^{47}$. La adherencia constituye una de las principales preocupaciones en el manejo ambulatorio de las enfermedades crónicas y otras condiciones de salud entre las que se reconoce la hipoacusia.

Intentando superar la crítica del paternalismo del cumplimiento terapéutico (o compliance $)^{48}$, concepto largamente utilizado desde la década de los setenta, la adherencia extiende la terapia más allá del acto médico, posibilitando que su abordaje considere la diversidad de prestaciones realizadas en los establecimientos sanitarios para el manejo de las enfermedades crónicas. Por otro lado, el "usuario" ya no espera pasivamente por una mejoría, más bien es reconocida su capacidad de aceptar o no las recomendaciones terapéuticas frente al manejo de su propia salud, lo que queda establecido en la adherencia por acordar las acciones a desarrollar en el tratamiento. Así, ya no se trata de instrucciones y obediencias enmarcadas en la pasividad de la "paciencia". Más bien se trata de un acto colaborativo para resolver un problema de salud que requiere de un tratamiento definido y acordado ${ }^{47}$.

El objetivo de lograr una buena adherencia terapéutica se basa en que mediante esto es posible alcanzar mejores resultados del tratamiento asignado para los usuarios en relación a la recuperación de su salud. Esto hace evidente 
la necesidad de contar con instrumentos de medición confiables para determinar los usuarios que están adhiriendo a su tratamiento y quienes no. Con respecto a la hipoacusia, es posible definir adherencia como el uso continuo de audífonos en personas diagnosticadas con esta condición, y que hayan acordado recibir y utilizar estos dispositivos de acuerdo a las recomendaciones entregadas por el equipo de salud. Esta conducta permitiría influir de forma positiva en la calidad de vida de las personas mejorando la comunicación oral y las relaciones sociales con su entorno.

A pesar de los desafíos metodológicos de las estimaciones porcentuales de adherencia al uso de audífonos, parece claro que una proporción de adultos mayores -en mayor o menor magnitud-finalmente abandona el uso de audífonos ${ }^{43}$. De las personas que requieren audífonos, solo un $25 \%$ los obtienen, y de estos, entre un $30 \%$ y un $50 \%$ no los utilizan ${ }^{8,39}$. La explicación a la subutilización de los audífonos se centra en la aceptación de la condición de salud (y, por ende, el uso del dispositivo) y en la adaptación a la prótesis auditiva, proceso de muchas dificultades y prejuicios que se relacionan con aspectos tanto técnicos y estéticos como sociales ${ }^{49}$. De los factores asociados a la baja adherencia en el uso de audífonos, destacan el efecto negativo del ruido ambiental, la poca comodidad, el aspecto poco estético de los audífonos, resultados iniciales decepcionantes, falta de motivación, dudas sobre funcionamiento, poca accesibilidad a servicio técnico, comentarios de otras personas y mala disposición previa, entre otros ${ }^{49,50}$. Menor relación tienen también las características como el nivel educacional o la polifarmacia ${ }^{20}$.

En los inicios del manejo de la hipoacusia en personas mayores, se asumió que actuando sobre la aferencia se podría corregir toda la vía auditiva. Sin embargo, actualmente se reconoce el rol del sistema nervioso central en la génesis de algunos tipos de presbiacusia ${ }^{50} \mathrm{y}$ de la función del sistema auditivo eferente en la progresión de ésta ${ }^{51}$. Los programas de rehabilitación auditiva tendrían una participación a nivel del sistema nervioso central, en el sentido de reeducar la audición, por lo que se intervendría más allá de solamente la aferencia ${ }^{52}$. El alto porcentaje de adultos mayores que no recibe beneficio con los audífonos y la complejidad de los aspectos anatomofisiológicos y cognitivos que llevan a las dificultades en la comunicación oral en las personas mayores, son factores que hacen necesaria la complementariedad del tratamiento de adaptación del uso de audífonos con programas de entrenamiento auditivo y/o rehabilitación auditiva centrada en estrategias comunicativas.

\section{Mejoras en la calidad de vida producto del uso de audífonos}

A partir de las consecuencias de la hipoacusia es posible reconocer qué intervenciones efectivas en usuarios con esta condición pueden mejorar de manera importante las relaciones sociales, emocionales, la comunicación, el funcionamiento cognitivo y la calidad de vida de las personas ${ }^{24}$.

La relación entre la hipoacusia y la calidad de vida se manifiesta desde reacciones emocionales (soledad, aislamiento, frustración, depresión, ansiedad y vergüenza, entre otros), de comportamiento (abandono de actividades, mayor dependencia) y cognitivas (confusión, dificultad en la concentración, distraibilidad y baja autoestima $)^{53}$. Es así como la evaluación del deterioro cognitivo debería ir acompañado de la evaluación y seguimiento de la calidad de $v \mathrm{da}^{54}$, considerando que solamente un $39 \%$ de las personas que padecen hipoacusia refieren tener una excelente calidad de vida global, comparado con el 68\% de la población sin hipoacusia. Las personas que padecen esta condición están generalmente menos satisfechas con su vida global que las personas sin hipoacusia ${ }^{53}$.

El aspecto psicológico, ambiental y social muestra una mejora global al comparar personas que usan audífonos versus las que no. Teniendo en cuenta que la comunicación es esencial para la vida y que la discapacidad auditiva hace que el proceso de comunicación se rompa, los procesos de rehabilitación que consideren los entornos sociales y familiares resultan fundamentales para que las personas retornen al mundo del sonido ${ }^{52}$.

Un antecedente que resulta interesante a considerar en rehabilitación, es que el aspecto social no necesariamente se ve mejorado en términos de la integración de las personas 
con hipoacusia a los entornos, puesto que para esto es necesario también modificar los hábitos sociales de aislamiento de las personas con hipoacusia ${ }^{55}$. Por otra parte, estudios señalan que el género muestra diferencias en los resultados de la rehabilitación sobre la calidad de vida $^{56}$. Las mujeres presentarían mejores resultados debido a que los hombres tienen más resistencia al uso de audífonos. Además, es el género femenino quien presenta una mayor inserción en la comunidad, aumentando así sus habilidades comunicativas, provocando una mejor inserción social y con esto una mejoría en la calidad de vida ${ }^{56}$.

Por su parte, los cuidadores de los pacientes -en su mayoría mujeres- con hipoacusia también se ven afectados en calidad de vida en el ámbito social, pero también psicológico, físico y medio ambiental ${ }^{56}$. La edad del cuidador también resulta importante, puesto que los más jóvenes se ven más afectados por el estrés y sobrecarga emocional del cuidado, además de la privación de las actividades y la vida social $^{56}$. Mientras que a mayor edad del cuidador menor impacto ya que estos presentan más experiencia de vida con una mayor capacidad de aceptación de los hechos.

Un correcto diagnóstico permite tener una orientación y rehabilitación adecuada a través de la generación de programas y estrategias enfocadas en la integración social, permitiendo que la calidad de vida se mantenga o mejore no solo en las personas con hipoacusia, sino que también para sus familiares y cuidadores. Por lo tanto, el uso de audífonos genera una mayor autonomía de la persona mayor, provocando que el cuidador se sienta más liberado, generando una mejoría en la calidad de vida de $\operatorname{ambos}^{57}$.

\section{El manejo de la hipoacusia en el contexto chileno}

Con el objetivo de ofrecer una atención de salud que garantice oportunidad, calidad y protección financiera para problemas de salud con alto impacto sanitario, y disminuir la brecha de oportunidad entre los sistemas público y privado, la última reforma de salud es considerada el mayor esfuerzo del sector en los últimos 25 años $^{58}$. Cubriendo tanto al sector público como el privado, el régimen de Garantías Explícitas en Salud (GES) incluye una selección de problemas sanitarios cuya priorización consideró principios como prevalencia, equidad y vulnerabilidad, lo que finalmente se tradujo en un listado limitado de enfermedades para cuyo tratamiento se contaba con suficiente capacidad de provisión en el país, especialmente en el sector público ${ }^{58}$. Asimismo, dichos tratamientos se tradujeron en el desarrollo de guías clínicas para cada problema de salud, documentos que sirven de recomendaciones y cuya característica principal fue su validación por parte de las sociedades científicas que agrupaban a los especialistas de cada área.

La hipoacusia bilateral fue uno de los 56 problemas incorporados al inicio del GES. Enfocado en personas de 65 años y más, su abordaje implicó la entrega de un audífono a todo quien cumpliera con los criterios diagnósticos ${ }^{19}$. A partir de esto, las prestaciones incluidas en el manejo de esta condición de salud fueron protocolizadas mediante el desarrollo de una guía clínica, la cual orientó los tiempos diagnósticos y de tratamiento para las personas diagnosticadas que se atendieran tanto en el sector público como privado de salud. Extrapolando datos de experiencias internacionales respecto al seguimiento de guías clínicas, y considerando que se trata de un documento con recomendaciones para los equipos médicos que atienden esta condición de salud, existe evidencia que indica que en ciertas circunstancias las guías clínicas no siempre son seguidas por los profesionales de la salud, por lo que existe una posibilidad que esto se replique en la realidad chilena ${ }^{25}$.

En su flujograma de seguimiento al usuario que recibe audífono, el documento establece el desarrollo de programa de consejería en rehabilitación audiológica, y de valoración y calibración del audífono en caso de ser necesario ${ }^{19}$, sin establecer de manera clara y explícita el tipo de intervención propuesta ni la modalidad de rehabilitación a seguir (individual o grupal). Lo anterior es relevante, ya que en Chile se ha establecido que cerca del $53 \%$ de las personas a las que se les prescribió el uso de audífonos no son adherentes a su tratamiento ${ }^{10}$, cifra con una amplia variabilidad de acuerdo al tipo de mediciones y usuarios, oscilando entre un 
$40 \%$ a un $80 \%$. Tales cifras dan cuenta de la importancia de determinar los motivos por los cuales las personas no usan los audífonos.

Un estudio internacional evaluó las diferencias en el aumento de la adherencia al uso de audífonos entre estrategias grupales e individuales, sin observar diferencias significativas respecto a los resultados en logros auditivos, la adherencia al audífono o las visitas no planificadas ${ }^{59}$. Otro estudio da cuenta de los beneficios indirectos de las intervenciones grupales al señalar que a través de éstas es posible mantener la calidad de la atención, puesto que permitiría destinar más horas clínicas a otras actividades, como el suministro de audífonos a un mayor número de usuarios, lo que reduciría los tiempos de espera y permite destinar mayor tiempo de atención a aquellos usuarios con necesidades de rehabilitación más complejas $^{60}$. Un estudio sobre el impacto del uso de audífonos en los beneficiaros del Fondo Nacional de Salud (FONASA) respecto al GES de "Hipoacusia bilateral en personas de 65 años y más que requieren uso de audífonos" señaló que el uso de estos dispositivos tiene efectos positivos en ciertos eventos que se busca evitar y que generan un impacto en la población de mayor edad, como el número de caídas y la presencia de mareos $^{61}$. La adecuación del diseño y la complejidad de las instrucciones serían determinantes en la continuidad en el uso de este dispositivo ${ }^{61}$, lo que puede repercutir en encontrar niveles subóptimos en el uso y necesidad de proponer intervenciones ${ }^{62}$.

\section{Prácticas en torno a la rehabilitación audiológica: influencia de la "Clasificación Internacional del Funcionamiento, de la Discapacidad y de la Salud" de la OMS}

Según Montano ${ }^{63}$, en un comienzo, los programas de rehabilitación para los pacientes con hipoacusia entregaban diversas prestaciones, dentro de las que se incluía no solamente la entrega de audífonos. Además, estaba la consejería, entrenamiento auditivo y lectura labial. Con el paso de los años el foco cambió hacia el diagnóstico, muy probablemente debido al desarrollo de nuevas tecnologías como lo fueron los exámenes objetivos y, en el caso de los audífonos, la incorporación de herramientas de análisis y calibración de circuitos de amplificación digital. De esta forma, la rehabilitación se orientó hacia la prescripción, calibración y evaluación de audífonos, de la mano con los avances en el área de la amplificación ${ }^{64}$. En esta época, se debe reconocer el esfuerzo realizado por la industria para adaptar las características acústicas de los audífonos a los requerimientos sugeridos por los hallazgos audiométricos. Como era de esperar, a medida que aumentó la disponibilidad de audífonos con mejores sistemas de amplificación y mayor rendimiento auditivo de éstos, existió un gradual declive en otras acciones de la rehabilitación "no tecnológicas". Existen otras razones que influyeron en la menor aplicación de programas de rehabilitación, entre las que se encontraría la mayor cantidad de tiempo que estas demandan $y$ el que solo recientemente se ha estudiado y demostrado su eficacia ${ }^{65}$.

Desde un comienzo la "Asociación Americana del Habla, Lenguaje y Audición” (ASHA $)^{66}$ reconoció que la intervención en pacientes con hipoacusia involucraba una amplia gama de acciones, las que van más allá de la evaluación y entrega de audífonos. Se reconoce la importancia de la consejería entregada al individuo y su familia, en relación al impacto de la pérdida auditiva. Dicha organización, a través de un comité especialmente formado, destacó la importancia del impacto de la pérdida auditiva en el funcionamiento dentro del contexto de la familia y ambiente, los que se transformaron en los principales objetivos de la intervención ${ }^{67}$. Autores como Erdman ${ }^{68}$ van más allá, denotando que la rehabilitación debería actuar sobre las consecuencias auditivas y no auditivas de la hipoacusia, resaltando con esto el rol de los aspectos emocionales y psicosociales.

En este cambio de énfasis influyó marcadamente la publicación por parte de la OMS de la "Clasificación Internacional del Funcionamiento, de la Discapacidad y de la Salud" (CIF) ${ }^{69}$, la cual fue la base de posteriores comisiones de trabajo de la ASHA. En éstas, la rehabilitación auditiva deja de referirse solo a procedimientos como el entrenamiento auditivo, la lectura labial o incluso el adaptar audífonos, reconociendo que es un proceso más amplio. Para la ASHA la rehabilitación es 
un proceso interactivo cuyo objetivo es facilitar las habilidades para minimizar o prevenir las limitaciones y restricciones que la hipoacusia puede producir en el bienestar y en la comunicación. La CIF influyó en la definición de la ASHA sobre rehabilitación auditiva, resaltando la importancia de los factores del contexto, tales como las influencias del medio ambiente, en la habilidad individual de funcionar con un impedimento ${ }^{65}$. Otros autores, como Boothroyd $^{70}$, plantean definiciones aún más en línea con la CIF, enfatizando que la rehabilitación actúa reduciendo las deficiencias en el funcionamiento, actividad, participación y calidad de vida.

Actualmente, gran parte de los adultos mayores con hipoacusia que reciben tratamiento son intervenidos mediante la implementación con audífonos, siendo limitada su participación en programas de rehabilitación ${ }^{71}$. Esto constituye un desafío tanto en las acciones de prevención de hipoacusia como en la implementación de tratamientos efectivos que mejoren la calidad de vida de las personas.

Existen dos modelos que han fundamentado la puesta en práctica de la rehabilitación auditiva. Por una parte, tenemos el "modelo biomédico" en que el clínico evalúa, realiza el diagnóstico, determinando y realizando las acciones terapéuticas necesarias ${ }^{65}$. En este modelo el rol del usuario es más bien pasivo, siendo dirigido por el clínico. Este sería el modelo base para la mayoría de los programas de intervención en que el énfasis está en determinar el grado, tipo de hipoacusia, las características que permiten la selección y calibración del audífono. Por otro lado, tenemos el "modelo de rehabilitación" en el cual los servicios entregados son más horizontales, interactivos y facilitadores. En éste, el clínico ayuda a identificar los problemas y trabaja junto con el usuario hacia una resolución, desempeñando el paciente un rol más activo en su propia rehabilitación. Se hace hincapié en el impacto de la pérdida auditiva en la función comunicativa dentro de un determinado contexto, un concepto muy ligado a la propuesta de la ICF de la OMS ${ }^{65}$. El modelo biopsicosocial sería la respuesta al modelo biomédico, dominante en las sociedades industrializadas de mediados del siglo $\mathrm{XX}^{72}$. Este enfoque biopsicosocial no sitúa la recuperación de la salud o la calidad de vida exclusivamente en los aspectos biológicos del deterioro auditivo -habitual en la práctica médica relativa a hipoacusia- que condiciona a la persona a un rol de "usuario" a la espera de su mejoría.

\section{Conclusión}

Considerando la revisión anterior, la implementación de un abordaje que busque tener un impacto positivo en la adherencia al uso de audífonos debe incluir y desarrollar programas de rehabilitación auditiva que consideren aspectos de contexto, facilitando el acceso a la rehabilitación a través de brindar prestaciones en aquellos puntos de la red que sean identificados como "de fácil acceso". Ejemplos de estos son juntas de vecinos o instituciones de la atención primaria de salud como centros de salud familiar (CESFAM), centros comunitarios de salud familiar (CECOF) o centros comunitarios de salud mental (COSAM), y/u otros escenarios que promuevan junto al entrenamiento comunicacional la participación en la red local, siguiendo los planteamientos desde un modelo de rehabilitación biopsicosocial de enfermedad y discapacidad. El brindar una atención de este tipo permitiría resaltar aspectos psicosociales compartidos por los usuarios, y potenciar herramientas de apoyo integral basadas en estrategias de solución de problemas, en técnicas específicas con evidencia probada en esta población, e incluir y priorizar la evaluación y promoción de los factores sociales (entorno familiar, redes de apoyo, ambientes saludables) relacionados a la hipoacusia en las personas mayores. Con esto se pretende mejorar la comprensión del fenómeno de la falta de adherencia al tratamiento, reconociendo en la persona junto con su entorno, un componente activo significativo en el autocuidado y participación en la resolución de problemas de salud. Reconocemos las limitaciones fácticas que plantea una rehabilitación de estas características en la red primaria de atención pudiendo significar mayor complejidad en la distribución y participación de recursos humanos, pero existen experiencias en otras patologías GES que han instaurado parte importante de la rehabilitación con este modelo. Se espera, además, 
mejorar la interacción entre los profesionales y los usuarios, otorgando herramientas a los equipos que ya no son solamente médicos, sino interdisciplinares, favoreciendo la comunicación de la red y reconociendo y abordando en terreno aspectos relevantes en la prevención. Así, el mejoramiento de la adherencia al uso de audífonos incentiva nuevas formas de organizar las acciones sanitarias en torno al manejo de la hipoacusia desde un enfoque de participación, tanto en la promoción de la salud como en la prevención de la enfermedad, modelo que a pesar de llevar tiempo implementado en el sistema público, permanece con desafíos latentes.

\section{Bibliografía}

1. United Nations, Department of Economic and Social Affairs, Population Division (2013). World Population Prospects: The 2012 Revision, Highlights and Advance Tables. Working Paper No. ESA/P/ WP.228.

2. Comisión Económica para América Latina y el Caribe (CEPAL), Observatorio Demográfico, 2013 (LC/G.2615-P), Santiago de Chile, 2014.

3. Salud Md. Estrategia Nacional de Salud para el cumplimiento de los Objetivos Sanitarios de la Década 2010-2020. Santiago de Chile: Ministerio de Salud; 2011.

4. Goycoolea MV. Editorial. Rev Méd Clín Las Condes. 2003;14:0.

5. Yamasoba T, Lin FR, Someya S, Kashio A, Sakamoto T, Kondo K. Current concepts in age-related hearing loss: epidemiology and mechanistic pathways. Hear Res. 2013;303:30-38.

6. Sprinzl GM, Riechelmann H. Current trends in treating hearing loss in elderly people: a review of the technology and treatment options - a mini-review. Gerontology. 2010;56:351-358.

7. Lee FS, Matthews LJ, Dubno JR, Mills JH. Longitudinal study of pure-tone thresholds in older persons. Ear Hear. 2005; 26:1-11.

8. Yueh B, Shapiro N, MacLean CH, Shekelle PG. Screening and management of adult hearing loss in primary care: scientific review. JAMA. 2003;289:19761985.

9. Guía Clínica para la Atención Primaria de las Personas Adultas Mayores. In: OPS/OMS, ed.; 2003.

10. Encuesta Nacional de Salud 2003. In: Salud Md, ed. Revisión actualizada el 23 de Abril del año 2019 ed; 2003.

11. Encuesta Nacional de Salud 2009. In: Salud Md, ed. Revisión actualizada el 25 de Abril del año 2019 ed; 2009.
12. Gratton MA, Vazquez AE. Age-related hearing loss: current research. Curr Opin Otolaryngol Head Neck Surg. 2003;11:367-371.

13. Van Eyken E, Van Camp G, Van Laer L. The complexity of age-related hearing impairment: contributing environmental and genetic factors. Audiol Neurootol. 2007;12:345-358.

14. Veras RP, Mattos LC. Audiology and aging: literature review and current horizons. Braz J Otorhinolaryngol. 2007;73:122-128.

15. Nabelek AK, Robinson PK. Monaural and binaural speech perception in reverberation for listeners of various ages. J Acoust Soc Am. 1982;71:1242-1248.

16. Chia EM, Wang JJ, Rochtchina E, Cumming RR, Newall P, Mitchell P. Hearing impairment and health-related quality of life: the Blue Mountains Hearing Study. Ear Hear. 2007;28:187-195.

17. Speech understanding and aging. Working Group on Speech Understanding and Aging. Committee on Hearing, Bioacoustics, and Biomechanics, Commission on Behavioral and Social Sciences and Education, National Research Council. J Acoust Soc Am. 1988;83:859-895.

18. Lin FR, Yaffe K, Xia J, et al. Hearing loss and cognitive decline in older adults. JAMA Intern Med. 2013; 173:293-299.

19. Ministerio de Salud, Chile. Guía Clínica "Hipoacusia bilateral en personas de 65 años y más que requieren uso de audífono”. Disponible en http://www.repositoriodigital.minsal.cl/ handle/2015/501.

20. Yueh B, Shapiro N, MacLean CH, Shekelle PG. Screening and management of adult hearing loss in primary care: scientific review. JAMA. 2003;289(15):1976-19785.

21. Mick P, Kawachi I, Lin FR. The association between hearing loss and social isolation in older adults. Otolaryngol Head Neck Surg. 2014;150(3):378384. doi:10.1177/0194599813518021.

22. Solheim J, Kværner KJ, Falkenberg E-S. Daily life consequences of hearing loss in the elderly. Disability and Rehabilitation. 2011; 33:2179-2185.

23. CEPAL. Panorama Social de América Latina 2012-Publicación de las Naciones Unidas-Santiago de Chile. CELADE 2012.

24. Mulrow CD, Aguilar C, Endicott JE, et al. Quality-oflife changes and hearing impairment. A randomized trial. Ann Intern Med. 1990;113:188-194.

25. Chisolm TH, Abrams HB, McArdle R. Shortand long-term outcomes of adult audiological rehabilitation. Ear Hear. 2004;25:464-477.

26. Hallam R, Ashton P, Sherbourne K, Gailey L. Acquired profound hearing loss: mental health and other characteristics of a large sample. Int J Audiol. 2006;45:715-723.

27. Nachtegaal J SJ, Smits C, Bezemer P, Van Beek J, Festen J, Kramer S. The Association Between Hearing 
Status and Psychosocial Health Before the Age of 70 Years: Results From an Internet-Based National Survey on Hearing. Ear Hear. 2009;30:302-312.

28. Mahapatra SB. Psychiatric and psychosomatic illness in the deaf. Br J Psychiatry. 1974; 125: 450-451.

29. Emmett SD, Francis HW. The Socioeconomic Impact of Hearing Loss in U.S. Adults. Otol Neurotol. 2015;36(3):545-550.

30. Solar O, Irwin A. A conceptual framework for action on the social determinants of health. Social Determinants of Health Discussion Paper 2 (Policy and Practice). World Health Organization. 2010.

31. Servicio Nacional de la Discapacidad (SENADIS). Primer Estudio Nacional de la Discapacidad. Santiago: Senadis. 2004. Disponible en: www.senadis. gob.cl/descarga/i/129/documento.

32. Larrañaga O. Inequality, Poverty and Social Policy: Recent Trends in Chile, OECD Social, Employment and Migration Working Papers, No. 85, OECD 2009.

33. Choi H, Burgard S, Elo IT, Heisler M. Are older adults living in more equal counties healthier than older adults living in more unequal counties? A propensity score matching approach. Soc Sci Med. 2015;141:82-90.

34. Informe Mundial sobre la Discapacidad. Geneve: Organización Mundial de la Salud; 2011.

35. McCormack A, Fortnum H. Why do people fitted with hearing aids not wear them? Int J Audiol. 2013;52:360-8.

36. Humes L. Issues in the assessment of auditory processing in older adults. In: Cacace At MD, ed. Controversies in Central Auditory Processing Disorder. San Diego: Plural Publishing 2008: 121-50.

37. Gates GA, Mills JH. Presbycusis. Lancet. 2005;366:1111-20.

38. Chang HP. Presbycusis among older Chinese people in Taipei, Taiwan: a Community-based study. Int J Audiol. 2007;46(12):738-45.

39. Dunlop RJ, Dennis KC, Gonzenbach SA, Abrams HB, Berardino JT, Styer SA, Hall A. Support personnel in VA audiology. Audiology Today. 2006;18(1):24-5.

40. Mener DJ, Betz J, Genther DJ, Chen D, Lin FR. Hearing loss and depression in older adults. J Am Geriatr Soc. 2013;61:1627-9.

41. McArdle R, Chisolm TH, Abrams HB, Wilson RH, Doyle PJ. The WHO-DAS II: measuring outcomes of hearing aid intervention for adults. Trends Amplif. 2005;9:127-143.

42. Chisolm TH, Johnson CE, Danhauer JL, et al. A systematic review of health-related quality of life and hearing aids: final report of the American Academy of Audiology Task Force On the Health-Related Quality of Life Benefits of Amplification in Adults. J Am Acad Audiol. 2007;18:151-183.

43. Popelka MM, Cruickshanks KJ, Wiley TL, Tweed TS, Klein BE, Klein R. Low prevalence of hearing aid use among older adults with hearing loss: the
Epidemiology of Hearing Loss Study. J Am Geriatr Soc. 1998;46:1075-1078.

44. Deafness and hearing impairment. Fact sheet No. 300. 2006. Disponible en http://www.who.int/ mediacentre/factsheets/fs300/en/index.html.

45. Bertoli S, Staehelin K, Zemp E, Schindler C, Bodmer D, Probst R. Survey on hearing aid use and satisfaction in Switzerland and their determinants. Int J Audiol. 2009;48:183-195.

46. Davis A, Smith P, Ferguson M, Stephens D, Gianopoulos I. Acceptability, benefit and costs of early screening for hearing disability: a study of potential screening tests and models. Health Technol Assess. 2007;11:1-294.

47. Organización Mundial de la Salud. Adherencia a los tratamientos a largo plazo. Pruebas para la acción; 2004.

48. Luftey K, Wishner W. Beyond "Compliance" Is "Adherence". Improving the prospect of diabetes care. Diabetes Care. 1999;22:635-639.

49. Pacala JT, Yueh B. Hearing deficits in the older patient: "I didn't notice anything". JAMA. 2012;307:1185-1194.

50. Gates GA. Central presbycusis: an emerging view. Otolaryngol Head Neck Surg. 2012;147:1-2.

51. Liberman MC, Liberman LD, Maison SF. Efferent feedback slows cochlear aging. J Neurosci. 2014;34:4599-4607.

52. Hickson L, Worrall, L., Scarinci, N. Active Communication Education (ACE). A program for older people with hearing impairment. In: Speechmark: Brackley ed. UK;2007.

53. Ciorba A, Bianchini C, Pelucchi S, Pastore A. The impact of hearing loss on the quality of life of elderly adults. Clin Interv Aging. 2012;7:159-163.

54. Dalton DS, Cruickshanks KJ, Klein BE, Klein R, Wiley TL, Nondahl DM. The impact of hearing loss on quality of life in older adults. Gerontologist. 2003;43:661-668.

55. Ribeiro Teixeira A, Gomes Almeida L, Pereira Jotz G, Cristine De Barba M. Qualidade de vida de adultos e idosos pós adaptação de próteses auditivas. Revista da Sociedade Brasileira de Fonoaudiologia. 2008; 13:357361.

56. Gassen Paulo M, Ribeiro Teixeira A, Pereira Jotz G, Cristine De Barba M, da Silva Bergmann R. Avaliação da Qualidade de Vida de Cuidadores de Idosos Portadores de Deficiência Auditiva: Influência do Uso de Próteses Auditivas. Arquivos Internacionais de Otorrinolaringologia. 2008;12:28-36.

57. Fonseca C, Oliveira L, Sérgio R, Carla N. Efeitos da adaptação às próteses auditivas na qualidade de vida, no equilíbrio e no medo de queda em idosos com perda neurossensorial. International Archives of Otorhinolaryngology. 2012;16:156-162.

58. Infante A, Paraje G. Reforma de Salud: Garantías Exigibles como Derecho Ciudadano. In: Larrañaga O, 
Contreras D, eds. Las Nuevas Políticas de Protección Social en Chile. Santiago de Chile: Uqbar Editores; 2010;77-118.

59. Collins MP, Liu CF, Taylor L, Souza PE, Yueh B. Hearing aid effectiveness after aural rehabilitation: individual versus group trial results. J Rehabil Res Dev 2013;50:585-598.

60. Sabate. Adherence to long-term therapies: policy for action. Meeting report, 4-5 June 2001. In: Organization. WHO/NMH/CCH, ed. Genove.; 2001. Disponible en https://apps.who.int/iris/ handle/10665/66984.

61. Bustamante M, Vidal C, López L. Impacto del Uso de Audífonos para Adultos Mayores en Chile. Información Tecnológica. 2014;25:177-84.

62. Maul X, Aracena K, Slater F, Breinbauer H. Adherencia y desempeño auditivo en uso de audífonos en pacientes adultos hipoacúsicos atendidos en la Red de Salud UC. Rev Otorrinolaringol Cir Cabeza Cuello. 2011;71:225-230.

63. Montano J, Spitzer J. (Eds.) Adult Audiologic Rehabilitation, Plural Publishing, San Diego, CA. 2009;25-36.

64. Hodgson WR. Hearing aid development and the role of audiology. En Hodgson WR(ed) Hearing aid assessment and use in audilogic rehabilitation $\left(3^{\mathrm{a}} \mathrm{ed}\right)$ Baltimore: Williams \& Wilkins. 1986. pp. 1-12.
65. Gagné JP, Jennings MB, Southall K. The international Classification of Functioning: Implications and Aplications to Audiologic Rehabilitation. En Montano J, Spitzer J. (Eds.) Adult Audiologic Rehabilitation, Plural Publishing, San Diego, CA. 2009;37-61.

66. ASHA. Definition of and competencies for aural rehabilitation. ASHA 1984;26(5):37-41.

67. ASHA. Knowledge and skills required for the practice of audiologist/aural rehabilitation. Asha. 2001. Vol 4.

68. Erdman SA. Counseling adults with hearing impairment. En Apiner J \& McCarthy P (Eds). Rehabilitative audiology: Children and adults ( $3^{\mathrm{a}} \mathrm{ed}$ ). Baltimore Williams \& Wilkins. 2000;435-472.

69. World Health Organization (WHO). International Classification of Functioning, Disability and Health: ICF. WHO 2001, ISBN 9241545429. Disponible en https://apps.who.int/iris/bitstream/ handle/10665/42407/9241545429.pdf.

70. Boothroyd A. Adult aural rehabilitation: what is it and does it work? Trends Amplif. 2007;11(2):63-71.

71. Mohr PE, Feldman JJ, Dunbar JL, et al. The societal costs of severe to profound hearing loss in the United States. Int J Technol Assess Health Care. 2000;16:112035.

72. Borrell F. El modelo biopsicosocial en evolución. Medicina Clínica. 2002; 119:175-179. 